\title{
Magnetic resonance imaging findings and prognosis of gastric-type mucinous adenocarcinoma (minimal deviation adenocarcinoma or adenoma malignum) of the uterine corpus: Two case reports
}

\author{
MAYO HINO $^{1}$, KEN YAMAGUCHI $^{1}$, KAORU ABIKO ${ }^{1}$, YUMIKO YOSHIOKA ${ }^{1}$, JUNZO HAMANISHI ${ }^{1}$, \\ EIJI KONDOH ${ }^{1}$, MASAFUMI KOSHIYAMA ${ }^{1}$, TSUKASA BABA ${ }^{1}$, NORIOMI MATSUMURA ${ }^{1}$, \\ SACHIKO MINAMIGUCHI ${ }^{2}$, AKI KIDO ${ }^{3}$ and IKUO KONISHI ${ }^{1}$
}

Departments of ${ }^{1}$ Gynecology and Obstetrics; ${ }^{2}$ Diagnostic Pathology; ${ }^{3}$ Diagnostic Imaging and Nuclear Medicine, Kyoto University Hospital, Kyoto, Kyoto 606-8507, Japan

Received July 7, 2015; Accepted January 20, 2016

DOI: $10.3892 / \mathrm{mco} .2016 .789$

\begin{abstract}
Our group previously documented the first, very rare case of primary gastric-type mucinous adenocarcinoma of the uterine corpus. Although this type of endometrial cancer appears to be similar to the gastric-type adenocarcinoma of the uterine cervix, its main symptoms, appearance on magnetic resonance imaging (MRI) and prognosis have not been fully elucidated due to its rarity. We herein describe an additional case of gastric-type mucinous adenocarcinoma of the endometrium and review the relevant literature. The two cases at our institution (Kyoto University Hospital, Kyoto, Japan) involved postmenopausal women with a primary complaint of abnormal genital bleeding. Microscopic examination of the hysterectomy specimens indicated a highly differentiated mucinous adenocarcinoma with a desmoplastic stromal reaction. Immunohistochemistry for HIK1083 and/or MUC6 was positive in both cases, suggesting a gastric phenotype. Both patients were diagnosed at an advanced stage, they relapsed or recurred immediately after adjuvant chemotherapy, and eventually succumbed to the disease. The main symptom of gastric-type mucinous adenocarcinoma of the uterine cervix is watery discharge, whereas abnormal genital bleeding in addition to watery discharge is mainly observed in the mucinous type of endometrial adenocarcinoma. Cystic cavities in the tumor are present on MRI in cases of endometrial origin, and prognosis is very poor due to resistance to chemotherapy. Thus, gastric-type mucinous adenocarcinoma of the uterine endometrium exhibits a clinical behavior that is similar to tumors originating from the uterine cervix, but is associated with distinguishing clinical
\end{abstract}

Correspondence to: Dr Ken Yamaguchi,Department of Gynecology and Obstetrics, Kyoto University Hospital, 54 Kawahara-Cho, Shogoin, Sakyo-Ku, Kyoto, Kyoto 606-8507, Japan

E-mail: soulken@kuhp.kyoto-u.ac.jp

Key words: endometrial mucinous adenocarcinoma, gastric-type, adenoma malignum, minimal deviation adenocarcinoma, uterine corpus symptoms. The incidence of gastric-type endometrial adenocarcinoma may be higher than expected.

\section{Introduction}

Mucinous adenocarcinoma of the uterine endometrium accounts for 1-9\% of all endometrial carcinomas (1). The diagnostic criteria of the World Health Organization require that $>50 \%$ of the neoplasm be composed of mucinous cells. The prognosis of these tumors tends to be relatively good (2). Mucinous endometrial carcinomas display a variety of morphological characteristics and may be subgrouped into endocervical and intestinal subtypes (1). Abiko et al, from our institution, documented the first case of mucinous endometrial adenocarcinoma with gastric-type histology (3). This histological subtype, however, is a rare malignant disease with only two case reports in the literature $(3,4)$. Thus, the clinical behavior of this rare disease has not been fully elucidated.

Gastric-type mucinous adenocarcinoma, also referred to as minimal deviation adenocarcinoma or adenoma malignum, accounts for $<1 \%$ of adenocarcinomas originating in the uterine cervix (5). Watery discharge is a major symptom of gastric-type adenocarcinoma of the uterine cervix, and macroscopic examination has revealed small cysts associated with the tumor. Although gastric-type mucinous adenocarcinoma of the uterine cervix exhibits a benign histological appearance, it is typically associated with chemoresistance and poor prognosis (6).

We herein describe an additional case of gastric-type mucinous endometrial adenocarcinoma treated at our institution. The clinical characteristics, symptoms, appearance on magnetic resonance imaging (MRI) and prognosis are described, along with a review of the relevant literature.

\section{Case reports}

Case 1. The first case involved a 72-year-old postmenopausal woman (gravida 4, para 2) presenting with a primary complaint of abnormal genital bleeding. The uterine cervical and endometrial cytology were class $\mathrm{V}$ and suspected endo- 
metrioid adenocarcinoma, respectively. Local examination indicated the presence of a dark bloody secretion from the external orifice of the uterus. Transvaginal ultrasonography revealed a walnut-sized tumor with an irregular shape in the uterine cavity. MRI demonstrated a 4-cm mass with low-intermediate intensity on T2-weighted images, and small cysts with high intensity, suggesting mucin elements, were identified in this tumor. The tumor invaded over half of the myometrium (Fig. 1A). Enlargement of a left obturator lymph node suggested the presence of metastasis. The serum levels of carcinoembryonic antigen (CEA) and carbohydrate antigen (CA)-125 were elevated $(5.5 \mathrm{ng} / \mathrm{ml}$, normal $<5 \mathrm{ng} / \mathrm{ml}$; and $40.3 \mathrm{U} / \mathrm{ml}$, normal $<35 \mathrm{U} / \mathrm{ml}$, respectively). Hysteroscopy revealed a polypoid tumor with atypical vessels in the uterine cavity. Endometrial biopsy revealed a well-differentiated mucin-producing adenocarcinoma, suggestive of gastric-type mucinous adenocarcinoma. The patient underwent semiradical hysterectomy (type II radical hysterectomy), bilateral salpingo-oophorectomy, pelvic lymphadenectomy, para-aortic lymphadenectomy and partial omentectomy. Intraoperative peritoneal washing cytology showed no evidence of malignant cells. Macroscopically, the tumor appeared to be whitish, sized $5.4 \mathrm{~cm}$ in the greatest dimension, arising from the left wall and invading more than half of the myometrium, without extension to the serosal surface (Fig. 1B). Microscopically, the tumor was diagnosed as a mucinous adenocarcinoma with well-formed glands, aggressively invading the myometrium, with a desmoplastic stromal reaction and a partial microcystic elongated and fragmented pattern (Fig. 1C). The carcinoma cells exhibited abundant pale cytoplasm and minimal nuclear atypia, with areas displaying fused glands and small clusters of cells with ovoid or irregular nuclei. Multiple pelvic and para-aortic lymph nodes metastases, as well as lymphovascular space invasion and nerve involvement, were observed. Immunohistochemistry showed positivity for MUC6, but negativity for HIK1083, p16 and p53 (Fig. 1D). The diagnosis was confirmed as gastric-type mucinous adenocarcinoma of the uterine corpus, pT1bN1M0, International Federation of Gynecology and Obstetrics (FIGO) stage IIIC2. Therefore, postoperative adjuvant chemotherapy with paclitaxel and carboplatin was administered; however, 2 months after adjuvant chemotherapy, recurrence at the para-aortic and pelvic lymph nodes was observed on a computed tomography (CT) scan. The cancer cells then spread to the lungs and the patient succumbed to the disease 6 months after recurrence to the para-aortic and pelvic lymph nodes.

Case 2. The second case of gastric-type mucinous adenocarcinoma of the uterine corpus in our institution has been previously reported (3). The patient was a 56-year-old postmenopausal woman with primary complaints of abnormal genital bleeding and vaginal discharge, who was diagnosed with high-grade adenocarcinoma of the uterine corpus by endometrial biopsy prior to surgery (3). Preoperative MRI revealed a solid tumor with multiple cysts exhibiting non-enhanced high intensity on T2-weighted images, suggesting mucin elements. The tumor invaded deep into the myometrium (Fig. 2A). A CT scan revealed para-aortic lymph node metastases and multiple small lung nodules, suggestive of lung metastases. The patient underwent total abdominal hysterectomy, bilateral salpingo-oophorectomy, pelvic lymphadenectomy, para-aortic lymphadenectomy and omentectomy. Gastric-type mucinous adenocarcinoma of the uterine corpus was diagnosed based on the pathological findings, which included well-formed glands composed of carcinoma cells with abundant clear or pale eosinophilic cytoplasm. Although nuclear anaplasia and architectural abnormalities were minimal, certain areas of the tumor displayed fused glands, carcinoma cells with nuclear atypia characterized by heterogeneous nuclear size and shape, and a desmoplastic stromal reaction. Endometrial hyperplasia was not observed. Immunohistochemistry was positive for MUC6, HIK1083, CA19-9 and CEA, but negative for MUC2, estrogen receptor, p16 and vimentin, suggesting a gastric phenotype (Fig. 2B). The pathological stage was pT1cN0M1. Although the patient underwent postoperative adjuvant chemotherapy with TS-1 and cisplatin (commonly used for gastric cancer), the disease progressed. The patient succumbed to the disease with peritonitis carcinomatosa and carcinomatous lymphangiomatosis 2 years and 4 months after surgery.

\section{Discussion}

Gastric-type mucinous adenocarcinoma is a newly characterized variant of mucinous adenocarcinoma with a gynecological origin. Gastric-type adenocarcinoma of the uterine cervix, also referred to as minimal deviation mucinous adenocarcinoma or adenoma malignum, represents $\sim 1-3 \%$ of all endocervical adenocarcinomas (6,7). Kojima et al (6) defined mucinous adenocarcinoma with gastric differentiation using the following criteria: i) Clear or pale eosinophilic cytoplasm, ii) voluminous cytoplasm and iii) distinct cell borders. The immunophenotype of these neoplasms is characterized by positivity for HIK1083 and/or MUC6, partial positivity for p53, and negativity for p16 (5). This type of adenocarcinoma of the uterine cervix is considered to be associated with poor prognosis due to its more aggressive clinical behavior compared with the usual types of adenocarcinoma of the uterine cervix $(6,8)$. However, among mucinous adenocarcinomas of the uterine corpus, only two cases displaying the gastric phenotype have been previously reported in the English literature $(3,4)$. Therefore, the biological characteristics of this type of tumor have not been fully elucidated, owing to its rarity.

The findings of the two previously reported cases, in addition to our case, are summarized in Table I. Gotoh et al (4) reported a case of synchronous mucinous adenocarcinoma of the endometrium and mucinous cystadenoma involving both ovaries in a 31-year-old woman with primary infertility. The three cases exhibited morphology, immunophenotype and clinical characteristics that are similar to those of gastric-type adenocarcinoma originating from the uterine cervix. All the patients presented with abnormal genital bleeding, and the primary complaint in case 2 in the present report involved vaginal discharge. The typical primary symptom of gastric-type adenocarcinoma of the uterine cervix is watery discharge, whereas gastric-type endometrial cancer may be suspected if abnormal genital bleeding is also a main symptom.

Kido et al (9) reported that tiny cysts associated with tumors were observed in $80 \%$ of the cases of gastric-type endocervical adenocarcinoma on MRI. The tumors in cases 1 and 2 displayed cystic regions on MRI, while in the case 
A
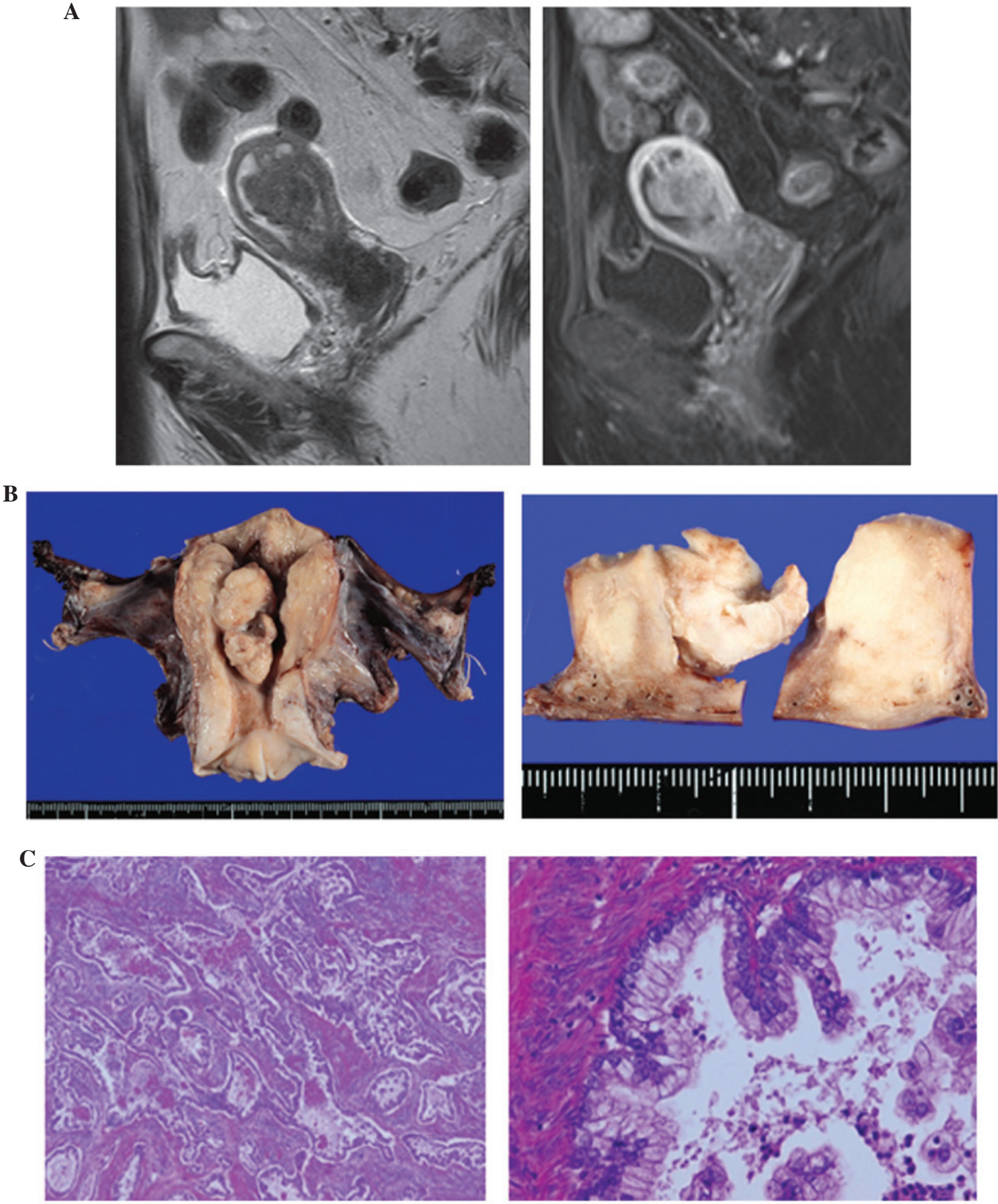

D
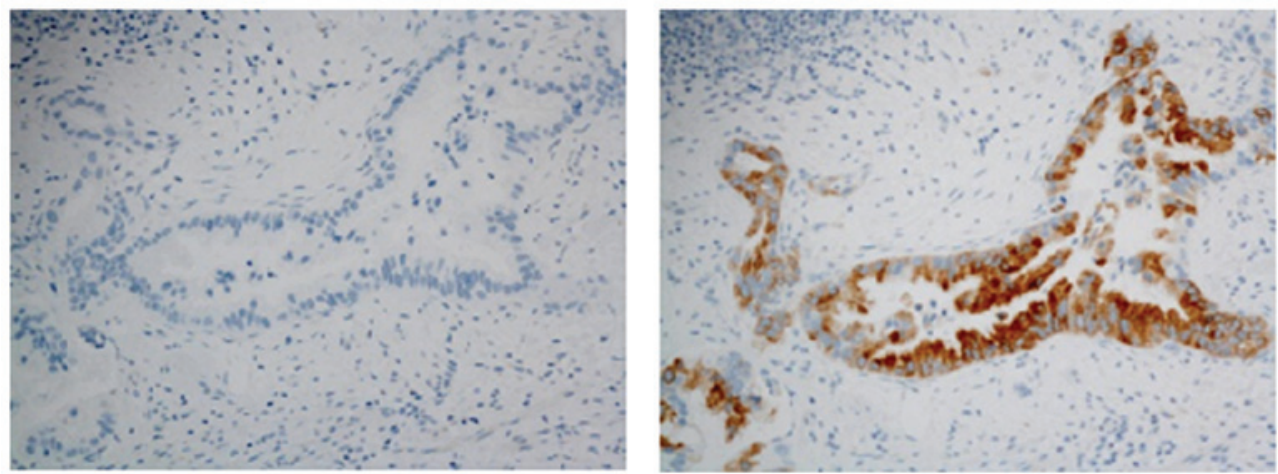

Figure 1. Magnetic resonance imaging (MRI) and pathological findings in case 1. (A) T2-weighted MRI scan showing a tumor with intermediate intensity (left panel). Enhanced T1-weighted images showing that the tumor invaded over half the thickness of the myometrium (right panel). (B) Macroscopically, the tumor was whitish, sized $5.4 \mathrm{~cm}$, arising from the left wall and invading over half of the myometrium. (C) The microscopic findings included a diffusely infiltrating mucinous adenocarcinoma with well-formed glands, aggressively invading the myometrium, along with a desmoplastic stromal reaction; (left panel) magnification, x100; right panel magnification, x400. (D) On immunohistochemistry, the tumor cells were positive for MUC6 (right panel), but negative for HIK1083 (left panel); magnification, $\mathrm{x} 400$ 


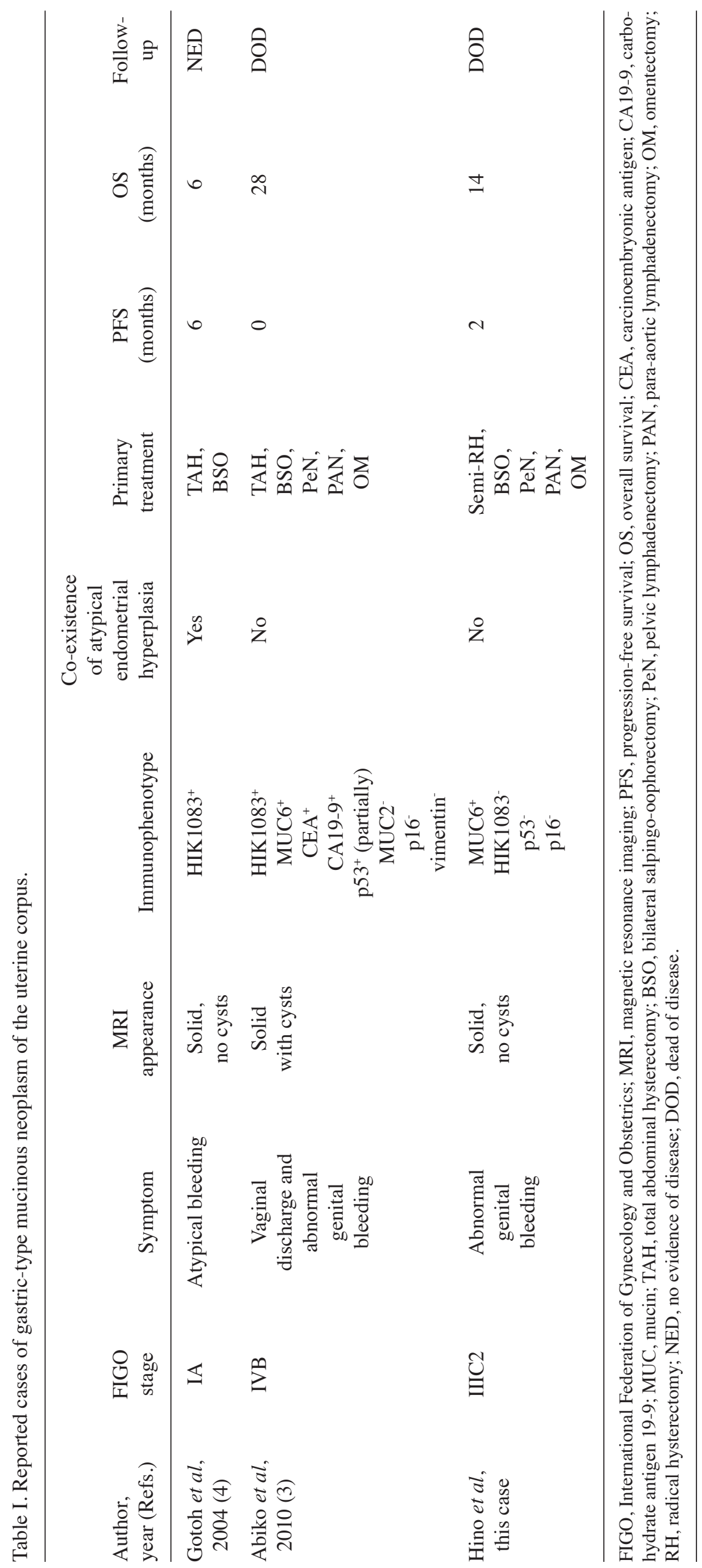


A
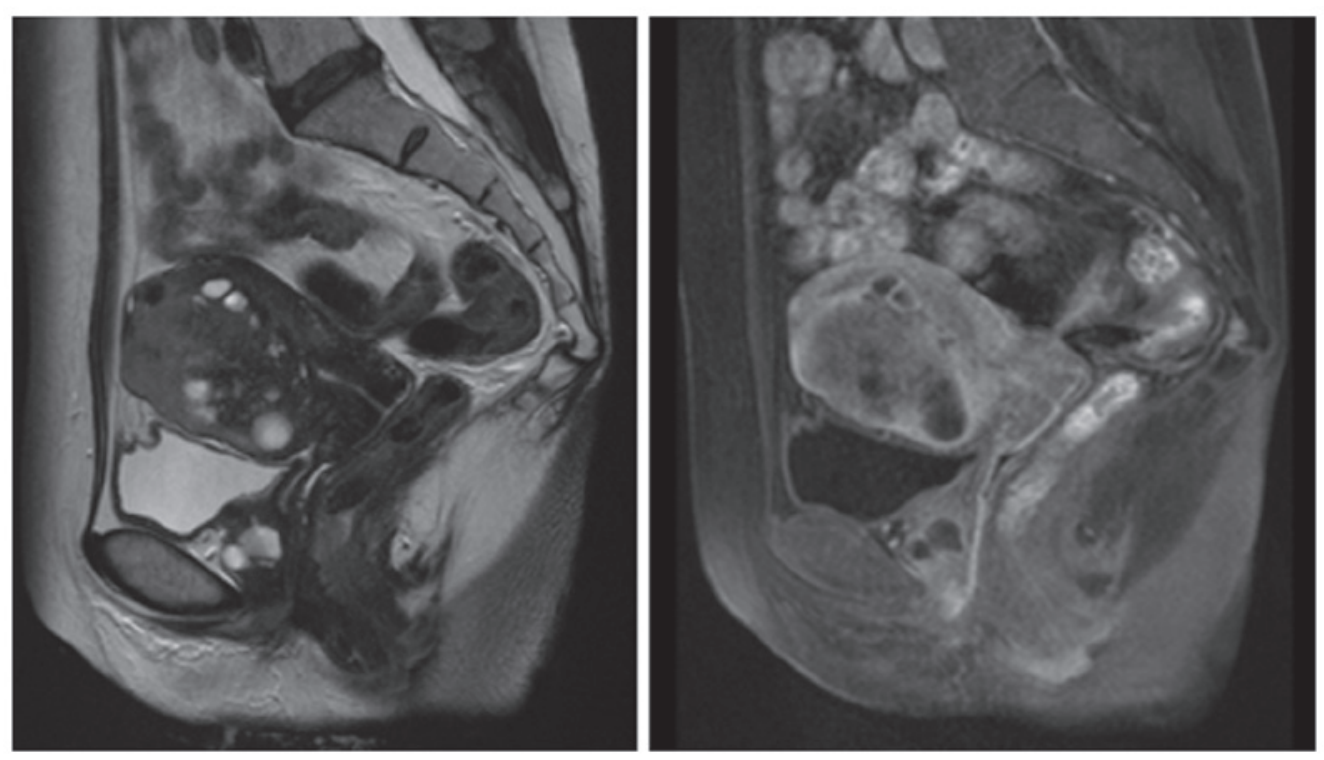

B
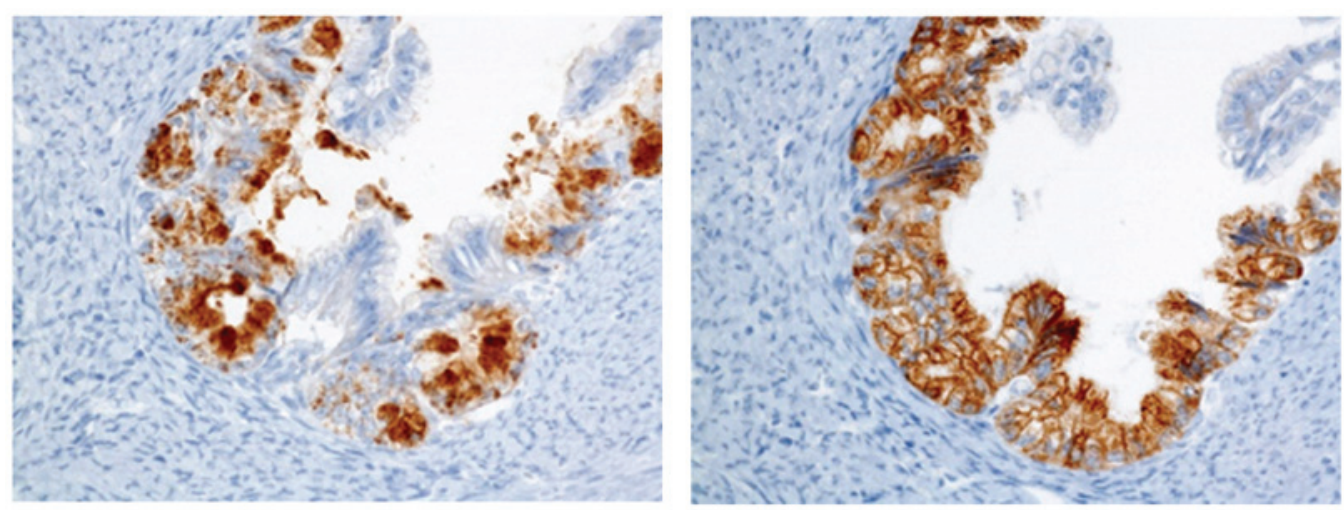

Figure 2. Magnetic resonance imaging (MRI) and immunohistochemical findings in case 2. (A) T2-weighted MRI scan showing a solid tumor with intermediate intensity, with high-intensity multiple cysts (left panel). An enhanced T1-weighted image revealed deep myometrial invasion (right panel). (B) Positive expression of HIK1083 (top) and MUC6 (bottom) in the cancer cells on immunohistochemistry; magnification, x400.

described by Gotoh et al (4), the small mass did not include cystic cavities. Thus, this type of endometrial cancer exhibits a cystic form in large tumors, such as gastric-type adenocarcinoma of the uterine cervix, when examined using MRI. However, cysts are not always present in small tumors. Histopathologically, the tumor is a low-grade adenocarcinoma, while immunohistochemistry for HIK1083 or MUC6 was positive in all three cases, indicating the gastric phenotype. There was no co-existing endometrial hyperplasia or endometrioid adenocarcinoma in our two cases, suggesting that these cancers were estrogen-independent. Case 2, which was FIGO stage IVB, presented with distant metastases at diagnosis, and the tumor was resistant to the chemotherapy commonly used for gastric cancer. Case 1, which was FIGO stage IIIC2, also exhibited chemoresistance to paclitaxel and carboplatin, which is generally an effective regimen for gynecological cancers. Both patients eventually succumbed to the disease.

In our institution, the 5-year overall survival rate of patients diagnosed with stage III endometrial cancer between 2004 and 2011 was $\sim 80 \%$ (unpublished data). The clinical behaviors of the cancer in our two cases indicates that gastric-type mucinous adenocarcinoma of the uterine endometrium exhibits an aggressive clinical behavior, even if the morphological findings are those of low-grade adenocarcinoma. The case reported by Gotoh et al, however, exhibited concomitant atypical endometrial hyperplasia and was stage IA (4). In that case, following complete resection, the prognosis was good, as the patient was reported to remain alive and cancer-free. Thus, gastric-type endometrial adenocarcinoma may be curable if complete resection of the tumor is feasible.

Nucci et al (10) suggested that architectural complexity alone is often the only diagnostic factor for endometrial mucinous adenocarcinoma, as several cases exhibit only mild-to-moderate nuclear atypia, thus highlighting the difficulty in differentially diagnosing gastric-type mucinous adenocarcinoma from the endocervical subtype. The reported frequency of mucinous endometrial adenocarcinoma that shows a relatively favorable prognosis ranges from $<1$ to $9 \%$ of all endometrial cancers $(1,11,12)$. As two cases of gastric-type endometrial adenocarcinoma were diagnosed in our institution over a period of 7 years, the frequency of gastric-type endometrial mucinous adenocarcinoma is possibly higher than expected. Although the new concept of gastric-type mucinous adenocarcinoma of the uterine corpus is not widely recognized, owing to its rarity among mucinous endometrial adenocarcinomas, gastric-type mucinous adenocarcinoma should be distinguished from other mucinous endometrial carcinomas, considering its aggressive clinical behavior. The 
diagnosis should be confirmed by immunohistochemistry in order to select the optimal treatment.

In summary, gastric-type adenocarcinoma of the uterine corpus is a relatively rare malignant disease. The incidence of gastric-type endometrial adenocarcinoma, however, is possibly higher than expected. The symptoms include abnormal genital bleeding and watery discharge. Gastric-type endometrial adenocarcinoma is identified as a solid, irregularly shaped tumor on MRI. Cases with large masses may display cystic cavities in the tumor, similar to gastric-type adenocarcinoma originating from the uterine cervix. These tumors exhibit an aggressive clinical behavior and poor prognosis, owing to chemoresistance. A better understanding of this disease is crucial in order to improve diagnosis and develop appropriate therapeutic strategies.

\section{References}

1. Ross JC, Eifel PJ, Cox RS, Kempson RL and Hendrickson MR: Primary mucinous adenocarcinoma of the endometrium. A clinicopathologic and histochemical study. Am J Surg Pathol 7: 715-729, 1983

2. Creasman WT, Morrow CP, Bundy BN, Homesley HD, Graham JE and Heller PB: Surgical pathologic spread patterns of endometrial cancer. A Gynecologic Oncology Group Study. Cancer 60 (Suppl 8): S2035-S2041, 1987.

3. Abiko K, Baba T, Ogawa M, Mikami Y, Koyama T, Mandai M and Konishi I: Minimal deviation mucinous adenocarcinoma ('adenoma malignum') of the uterine corpus. Pathol Int 60: 42-47, 2010.

4. Gotoh T, Hayashi N, Takeda S, Itoyama S, Takano M and Kikuchi Y: Synchronous mucinous adenocarcinoma of the endometrium and mucinous cystadenoma of bilateral ovaries presenting during fertility therapy. Int $\mathrm{J}$ Gynecol Cancer 14: 169-171, 2004.
5. Mikami Y and McCluggage WG: Endocervical glandular lesions exhibiting gastric differentiation: An emerging spectrum of benign, premalignant and malignant lesions. Adv Anat Pathol 20: 227-237, 2013.

6. Kojima A, Mikami Y, Sudo T, Yamaguchi S, Kusanagi Y, Ito M and Nishimura R: Gastric morphology and immunophenotype predict poor outcome in mucinous adenocarcinoma of the uterine cervix. Am J Surg Pathol 31: 664-672, 2007.

7. Silverberg SG and Hurt WG: Minimal deviation adenocarcinoma ('adenoma malignum') of the cervix: A reappraisal. Am J Obstet Gynecol 121: 971-975, 1975.

8. Gilks CB, Young RH, Aguirre P, DeLellis RA and Scully RE: Adenoma malignum (minimal deviation adenocarcinoma) of the uterine cervix. A clinicopathological and immunohistochemical analysis of 26 cases. Am J Surg Pathol 13: 717-729, 1989.

9. Kido A, Mikami Y, Koyama T, Kataoka M, Shitano F, Konishi I and Togashi K: Magnetic resonance appearance of gastric-type adenocarcinoma of the uterine cervix in comparison with that of usual-type endocervical adenocarcinoma: A pitfall of newly described unusual subtype of endocervical adenocarcinoma. Int J Gynecol Cancer 24: 1474-1479, 2014.

10. Nucci MR, Prasad CJ, Crum CP and Mutter GL: Mucinous endometrial epithelial proliferations: A morphologic spectrum of changes with diverse clinical significance. Mod Pathol 12: 1137-1142, 1999.

11. Fujiwara $\mathrm{M}$ and Longacre TA: Low-grade mucinous adenocarcinoma of the uterine corpus: A rare and deceptively bland form of endometrial carcinoma. Am J Surg Pathol 35: 537-544, 2011.

12. Plataniotis G and Castiglione M; ESMO Guidelines Working Group: Endometrial cancer: ESMO Clinical Practice Guidelines for diagnosis, treatment and follow-up. Ann Oncol 21 (Suppl 5): $\mathrm{v} 41-\mathrm{v} 45,2010$. 